\title{
Microorganisms Isolated From the Bile of the Patients who have Undergone Cholecystectomy and Their Antibiotic Resistance Pattern: Multicenter Prospective Study
}

\section{Derya Ozturk-Engin ( $\sim$ dr.deryaengin@gmail.com )}

Fatih Sultan Mehmet Training and Research Hospital: Fatih Sultan Mehmet Egitim ve Arastirma Hastanesi https://orcid.org/0000-0002-2566-7317

\section{Canan Agalar}

Fatih Sultan Mehmet Training and Research Hospital: Fatih Sultan Mehmet Egitim ve Arastirma Hastanesi

\section{Cag Yasemin}

Medeniyet University Goztepe Training and Research Hospital: Medeniyet Universitesi Goztepe Egitim ve Arastirma Hastanesi

\section{Fatma Kesmez-Can}

Atatürk Üniversitesi Tıp Fakültesi: Ataturk Universitesi Tip Fakultesi

ilker İnanç Balkan

Cerrahpasa Medical School: Istanbul Universitesi-Cerrahpasa Cerrahpasa Tip Fakultesi

\section{Oguz Karabay}

Sakarya Üniversitesi: Sakarya Universitesi

\section{Seniha Senbayrak}

Haydarpasa Training and Research Hospital: Istanbul Haydarpasa Numune Egitim ve Arastirma Hastanesi

\section{Busra Meral Cetinkaya}

Fatih Sultan Mehmet Training and Research Hospital: Fatih Sultan Mehmet Egitim ve Arastirma Hastanesi

\section{Mehmet Timucin Aydın}

Fatih Sultan Mehmet Training and Research Hospital: Fatih Sultan Mehmet Egitim ve Arastirma Hastanesi

\section{Kadir Tomas}

Rize Üniversitesi: Recep Tayyip Erdogan Universitesi

\section{Esra Disci}

Ataturk University: Ataturk Universitesi

\section{Ali Surmelioglu}


Haydarpasa Training and Research Hospital: Istanbul Haydarpasa Numune Egitim ve Arastirma Hastanesi

\section{Orhan Alimoglu}

Goztepe Training and Research Hospital: Medeniyet Universitesi Goztepe Egitim ve Arastirma Hastanesi

\section{Ozgur Ekinci}

Goztepe Training and Research Hospital: Medeniyet Universitesi Goztepe Egitim ve Arastirma Hastanesi

\section{Emrah Akın}

Sakarya University: Sakarya Universitesi

\section{Mehmet Koroglu}

Sakarya University: Sakarya Universitesi

\section{Mehmet Velidedeoglu}

Cerrahpasa Medical School: Istanbul Universitesi-Cerrahpasa Cerrahpasa Tip Fakultesi

\section{Handan Ankaralı}

Goztepe Training and Research Hospital: Medeniyet Universitesi Goztepe Egitim ve Arastirma Hastanesi

\section{Esra Kocoğlu}

Goztepe Training and Research Hospital: Medeniyet Universitesi Goztepe Egitim ve Arastirma Hastanesi

\section{Mirkhaliq Javadov}

Yeditepe University: Yeditepe Universitesi

\section{Berrin Papila-Kundaktepe}

Cerrahpasa Medical School: Istanbul Universitesi-Cerrahpasa Cerrahpasa Tip Fakultesi

\section{Naz Oguzoglu}

Haydarpasa Training and Research Hospital: Istanbul Haydarpasa Numune Egitim ve Arastirma Hastanesi

\section{Erkan Ozmen}

Ataturk University: Ataturk Universitesi

\section{Ramazan Donmez}

Yeditepe University: Yeditepe Universitesi

\section{Ertunc Mega}

Fatih Sultan Mehmet Training and Research Hospital: Fatih Sultan Mehmet Egitim ve Arastirma Hastanesi

\section{Sebahat Aksaray}

Haydarpasa Training and Research Hospital: Istanbul Haydarpasa Numune Egitim ve Arastirma Hastanesi

\section{Fatih Agalar}

Yeditepe University: Yeditepe Universitesi

\section{Research Article}

Keywords: Cholecystectomy, Bile culture, Antimicrobial Resistance 
Posted Date: February 28th, 2022

DOI: https://doi.org/10.21203/rs.3.rs-1370594/v1

License: (c) (i) This work is licensed under a Creative Commons Attribution 4.0 International License. Read Full License 


\section{Microorganisms Isolated From the Bile of the Patients who have Undergone Cholecystectomy and Their Antibiotic Resistance Pattern: Multicenter Prospective Study}

Ozturk- Engin D et al. Bile culture in patients undergoing cholecystectomy

Ozturk-Engin D, Agalar C, Cag Y, Kesmez-Can F, Balkan II, Karabay O, Senbayrak S, MeralCetinkaya B, Aydın MT, Tomas K, Disci E, Surmelioglu A, Alimoglu O, Ekinci O, Akın E, Köroğlu M, Velidedeoglu M, Ankaralı H, Kocoglu E, Javadov M, Papilla-Kundaktepe B, Oguzoglu N, Ozmen E, Donmez R, Mega E, Aksaray S, Agalar F

Derya Ozturk-Engin, Department of Infectious Diseases and Clinical Microbiology. Health Sciences University, Fatih Sultan Mehmet Training and Research Hospital, Atasehir/ Istanbul, Turkey.

Canan Agalar, Department of Infectious Diseases and Clinical Microbiology. Health Sciences University, Fatih Sultan Mehmet Training and Research Hospital, Atasehir/ Istanbul, Turkey.

Yasemin Cag, Department of Infectious Diseases and Clinical Microbiology. Medeniyet University, Göztepe Training and Research Hospital, Kadikoy/Istanbul,Turkey.

Fatma Kesmez Can, Department of Infectious Diseases and Clinical Microbiology. Ataturk University, Faculty of Medicine, Yakutiye/Erzurum, Turkey.

Ilker Inanc Balkan, Department of Infectious Diseases and Clinical Microbiology. Istanbul University-Cerrahpasa, Cerrahpasa Medical School, Fatih/Istanbul, Turkey.

Oguz Karabay, Department of Infectious Diseases and Clinical Microbiology. Sakarya University, Faculty of Medicine. Serdivan/Sakarya.Turkey.

Seniha Senbayrak, Department of Infectious Diseases and Clinical Microbiology, Health Sciences University, Haydarpasa Numune Training and Research Hospital, Uskudar/ Istanbul,Turkey 
Busra Meral Çetinkaya, Department of Infectious Diseases and Clinical Microbiology, Health Sciences University, Fatih Sultan Mehmet Training and Research Hospital, Atasehir/ Istanbul. Turkey.

Mehmet Timuçin Aydın Department of General Surgery, Health Sciences University, Fatih Sultan Mehmet Training and Research Hospital, Atasehir/ Istanbul, Turkey.

Kadir Tomas, Department of General Surgery. Recep Tayyip Erdogan University, Faculty of Medicine, Rize. Turkey.

Esra Disci, Department of General Surgery. Ataturk University, Faculty of Medicine, Yakutiye/ Erzurum. Turkey.

Ali Surmelioglu, Department of General Surgery, Health Sciences University, Haydarpasa Numune Training and Research Hospital, Uskudar/ Istanbul,Turkey.

Orhan Alimoglu, Department of General Surgery. Medeniyet University, Göztepe Training and Research Hospital, Kadikoy/Istanbul,Turkey.

Ozgur Ekinci, Department of General Surgery. Medeniyet University, Göztepe Training and Research Hospital, Kadikoy/Istanbul,Turkey.

Emrah Akın, Department of General Surgery. Sakarya University, Faculty of Medicine. Serdivan/Sakarya.Turkey.

Mehmet Köroglu, Department of Medical Microbiology. Sakarya University, Faculty of Medicine Serdivan/Sakarya. Turkey.

Mehmet Velidedeoglu, Department of General Surgery. Istanbul Univercity-Cerrahpasa, Cerrahpasa Faculty of Medicine, Fatih/Istanbul, Turkey.

Handan Ankaralı, Department of Biostatistics. Medeniyet University, Göztepe Training and Research Hospital, Kadikoy/Istanbul,Turkey.

Esra Kocoglu, Department of Medical Microbiology, Istanbul Medeniyet University Faculty of Medicine, Istanbul. Turkey.

Mirkhaliq Javadov, Department of General Surgery. Yeditepe Universtiy, Faculty of Medicine, Istanbul,Turkey. 
Berrin Papilla-Kundaktepe, Department of General Surgery. Istanbul Univercity-Cerahpasa, Cerrahpasa Faculty of Medicine, Fatih/Istanbul. Turkey

Naz Oguzoglu, Department of Medical Microbiology. Health Sciences University, Haydarpasa Numune Training and Research Hospital, Uskudar/ Istanbul,Turkey.

Erkan Ozmen, Department of Medical Microbiology. Ataturk University, Faculty of Medicine, Yakutiye/Erzurum.Turkey.

Ramazan Donmez, Department of General Surgery. Yeditepe Universtiy, Faculty of Medicine, Istanbul,Turkey.

Ertunç Mega, Department of Surgery. Health Sciences University, Fatih Sultan Mehmet Training and Research Hospital, Atasehir/ Istanbul, Turkey.

Sebahat Aksaray, Department of Medical Microbiology. Health Sciences

University,Haydarpasa Numune Training and Research Hospital, Uskudar/ Istanbul,Turkey.

Fatih Agalar, Department of General Surgery. Yeditepe Universtiy, Faculty of Medicine, Istanbul,Turkey.

Corresponding author: Derya Ozturk-Engin, MD, Professor, Department of Infectious Diseases and Clinical Microbiology. Health Sciences University, Fatih Sultan Mehmet Training and Research Hospital, Atasehir/ Istanbul. Turkey.

Telephone number: +90505 7512655

Email address: derya.ozturkengin@ @bu.edu.tr; dr.deryaengin@gmail.com.

Our study was designed prospectively and approval was obtained from the local ethics committee before the study started. The ethics committee document obtained from the ethics committee is attached. Ethics committee document is written in Turkish. However, if requested, we can send it in English. In our study, samples taken peroperatively were examined. 


\begin{abstract}
Background Gallbladder and biliary tract infections are diseases with high mortality rates if they are not treated properly. Microbiological evaluation of perioperatively collected samples both ensures proper treatment of patients and guides empirical treatment due to the determination of microorganism susceptibility.
\end{abstract}

Aims This study aimed to isolate the microorganisms in bile cultures from patients who underwent cholecystectomy and to determine sensitivity results of these microorganisms.

Methods This study was a multi-center and prospective design, included 360 patients, and was performed between 2019 and 2020. Culture results of bile taken during cholecystectomy were evaluated.

Results Bacterial growth was found in the bile cultures of 84 out of 360 (23.3\%) patients. Patients were divided into two groups according to whether they had risk factors for resistant microorganisms or not. While Escherichia coli $(\mathrm{n}=11,13 \%)$, Enterococcus $\mathrm{spp} .(\mathrm{n}=8,9.5 \%)$, and Enterobacter spp. $(\mathrm{n}=4,4.7 \%)$ were detected most frequently in patients without risk. Staphylococcus spp. $(\mathrm{n}=17,20.2 \%)$, Enterococcus spp., $(\mathrm{n}=16,19 \%)$, and E. coli $(\mathrm{n}=8$, 9.5\%) were the most frequently found microorganism in risky patients. Bile culture positivity was higher in males $(p=0.001),>60$ years $(p=0.010)$, history of biliary disease $(p=0.002)$, underwent biliary surgery before cholecystectomy $(\mathrm{p}=0.002)$, having biliary instrumentation $(p=0.014)$, operation performed concurrently with a cholecystectomy $(p=0.001)$, and presence of complications $(\mathrm{p}=0.001)$.

Conclusions Our study shows that when starting empirical antibiotic treatment for bile ducts, whether patients are at risk for the development of resistant bacterial infection should be evaluated after which antibiotic selection should be made accordingly.

Key words: Cholecystectomy, Bile culture, Antimicrobial Resistance 


\section{Introduction}

Gallbladder and biliary tract infections are an important health problem. Obstruction of the bile flow is the most important cause of biliary tract infections. Obstruction causes an increase in pressure in the bile duct, and bacteria can reach the systemic circulation through hepatic sinusoids when there is high pressure [1]. Although the most important reason for obstruction of bile flow is gallstones, tumors originating from surrounding structures and surgical injury to the bile ducts can also cause obstruction in the biliary tract [2].

Some experts have stated that the presence of microorganisms in the bile does not always indicate an active infection [3]. However, the detection of bactobilia during biliary surgery not only serves as a guide for appropriate antibiotic prophylaxis but also for administration of empirical therapy and changes in antimicrobial therapy when necessary. Bactobilia is common in patients with risk factors, such as advanced age, biliary obstruction, previous biliary instrumentation, acute cholecystitis, common bile duct stones, cholangitis and nonfunctioning gallbladder, previous biliary sepsis, bilioenteric anastomosis, and suppressed immune status $[4,5]$.

Gallbladder and biliary tract infections are diseases with high mortality rates if they are not treated properly. In patients with moderate or severe acute cholecystitis, antimicrobial therapy is recommended, and this therapy should be chosen considering the local antimicrobial resistance properties of microorganisms [6]. Limited information about the infectious agents in the bile ducts and their resistance patterns exists. This multicenter prospective study was designed to evaluate microorganisms with their corresponding resistance patterns that have been isolated from the bile of patients who have undergone cholecystectomy.

\section{Methods}

\section{Study design}

This multicenter and prospective study was performed in patients undergoing cholecystectomy between January 2019 and April 2020. Eight centers were included in the study. Consecutive patients were selected to participate in this study. The Ethical Committee of the Istanbul Fatih Sultan Mehmet Training and Research Hospital, Istanbul, Turkey, approved the study (FSMEAH-KAEK- 2020/11), and informed consent was obtained from all participants. 


\section{Inclusion criteria}

Patients aged >17 who were scheduled for cholecystectomy were included.

\section{Data collection}

Bile culture Recorded data included age, gender, body mass index (BMI), comorbidities, history of hospitalizations within the last year, biliary surgery history, history of biliary disease, presence of biliary instrumentation, history of antibiotic use in the last three months, symptoms of patients, American Society of Anaesthesiologists (ASA) score, the presence of operation concurrently with cholecystectomy, complications, laboratory and culture results, and patterns of antimicrobial in the microorganisms.

During cholecystectomy, 5-10 $\mathrm{ml}$ of bile fluid was aspirated under sterile conditions and/or a $1.5 \mathrm{~cm}^{2}$ biopsy sample was taken from the bile mucosa. The sample was placed in a sterile tube containing sterile saline. Bile fluid and mucosal samples were sent to the microbiology laboratory for cultivation on culture plates. Chocolate and MacConkey agars and aerobic and anaerobic blood culture media were used for cultures. The media were incubated at 35 to $37^{\circ} \mathrm{C}$. Aerobic media were kept for 18 to 24 and anaerobic media for 48 to $96 \mathrm{~h}$. Growing strains were identified by conventional methods, and VITEK $2 \AA$ Compact (Biomerieux. France) and/or matrix-assisted laser desorption ionization time-of-flight mass spectrometry (MALDI-TOF MS). VITEK-2 ${ }^{\circledR}$ Compact automated systems were used to study antimicrobial susceptibility of microorganisms. The antibiogram data were analyzed according to European Committee on Antimicrobial Susceptibility Testing (EUCAST) recommendations [7].

\section{Definitions}

BMI was detected according to CDC. (https://www.cdc.gov/healthyweight/assessing/bmi/ adult bmi/index.html).

Risk factors Diabetes mellitus (DM), malignicies, immunosuppression, biliary disease, biliary surgery, biliary instrumentation, antibiotic use within the last three months, and /or hospitalization during the previous year were identified as the risk factors $[4,8,9]$.

Acute cholecystitis Inflammation of the gallbladder with pain in the right upper quadrant [10]. 


\section{Statistical analysis}

The SPSS (version 23) program was used for statistical analysis. Descriptive statistics of the data are presented in tables as mean, standard deviation (SD), median, number, and \% frequencies. The compliance of numerical data to the normal distribution was performed using the Shapiro-Wilk test. It was determined that numerical variables did not show normal distribution. The relationships between the presence of risk and other characteristics of the patients were evaluated using the Pearson's chi-square analysis and the Mann-Whitney U test depending on the type and distribution of variables. $\mathrm{P}<0.05$ was accepted as the statistical significance level.

\section{Results}

Gallbladder samples from 360 patients were taken during cholecystectomy. Most the patients who participated in the study were women $(n=247,68.6 \%)$. Mean age was $51.1 \pm 13.2$ years. The duration of patients' symptoms was $136.4 \pm 212.2($ median $=60 ; \min -\max =1$ and 1470) days.

Laparoscopic surgery was more frequently performed $(n=330,91.6 \%)$ compared to open surgery $(n=23,6.3 \%)$. An operation that started laparoscopically was converted to open surgery for seven patients (1.9\%). Culture positivity was higher in patients with acute cholecystitis than in those patients with symptomatic cholelithiasis $(\mathrm{P}<0.001)$.

Indications for cholecystectomy were symptomatic cholelithiasis in 328 patients (91.1\%), acute cholecystitis in 71 patients $(19.7 \%)$, acute pancreatitis in seven patients $(1.9 \%)$, biliary polyps in three patients $(0.8 \%)$, biliary and pancreatic malignant tumors, perforation in the gall bladder, and cytoreductive surgery in two patients $(0.5 \%)$. Other indications were present in four patients (1.1\%). Fifty-five patients (15.2\%) underwent surgery for both symptomatic cholelithiasis and acute cholecystitis. Among the 71 patients with acute cholecystitis, 27 (38\%) had biliary disease, five (7\%) had a history of biliary surgery, and one (1.4\%) had a history of biliary instrumentation and sixty-three $(88.7 \%)$ patients underwent surgery while under treatment and eight (11.2\%) patient underwent surgery with surgical prophylaxis.

One hundred sixty-five patients $(45.8 \%)$ had underlying comorbid conditions. Hypertension and DM were the most common underlying conditions. Characteristics of the patients are presented in Table 1. 


\section{Culture Results}

Seventy-five patients $(22.5 \%)$ of the patients used antibiotics before biliary culture. From a total of 84 patients (23.3\%), 105 strains were grown. The most frequently isolated bacteria were Enterococcus spp. $(\mathrm{n}=24,22.8 \%)$, Staphylococcus $\mathrm{spp} .(\mathrm{n}=20,19 \%)$, E. coli $(\mathrm{n}=19,18.1 \%)$, Klebsiella spp. $(\mathrm{n}=10,9.5 \%)$, Streptococci $\mathrm{spp} .(\mathrm{n}=8,7.6 \%)$, and Enterobacter $\mathrm{spp} .(\mathrm{n}=7$, $6.6 \%)$.

The factors affecting culture positivity in the patients undergoing cholecystectomy were male gender $(p=0.001),>60$ years of age $(p=0.010)$, history of biliary disease $(p=0.002)$, underwent biliary surgery before cholecystectomy $(\mathrm{p}=0.002)$, having biliary instrumentation $(p=0.014)$, operation occurred concurrently with cholecystectomy $(p=0.001)$, and/or presence of complications $(\mathrm{p}=0.001)$. Because only two patients with a BMI $<19$ were enrolled in the study, the BMI result was not considered significant. (Table 2).

Leukocyte count $(\mathrm{p}=0.017)$, polymorphonuclear leukocytes $(\mathrm{PNL})$ ratio $(\mathrm{p}=0.001)$, C-reactive protein $(\mathrm{CRP})(\mathrm{p}=0.019)$, creatinine $(\mathrm{p}=0.008)$, alkaline phosphatase (ALP) $(\mathrm{p}=$ $0.010)$, total bilirubin $(\mathrm{p}=0.003)$, and glucose $(\mathrm{p}=0.003)$ values were found to be statistically significantly higher in patients with growth in the bile culture (Table 3 ).

The patients were divided into two groups according to their risk factors. E. coli $(\mathrm{n}=$ 11, 1\%), Enterococcus spp. $(\mathrm{n}=8,9.5 \%)$ and Enterobacter spp. $(\mathrm{n}=4,4.7 \%)$ were the most frequently found bacteria in patients without any risk factors. Staphylococcus spp. $(\mathrm{n}=17$, $20.2 \%)$, Enterococcus spp., $(\mathrm{n}=16,19 \%)$, and $E$. coli $(\mathrm{n}=8,9.5 \%)$ were the most frequently found bacteria in risky patients.

In the no-risk patients, the sensitivity of cefuroxime axetil was determined as $33 \%$, amoxicillin-clavulanate 54\%, ceftriaxone, ceftazidime, and piperacilline-tazobactam $60 \%$ each, cefepime $73 \%$, trimethoprim-sulfamethoxazole $80 \%$, gentamycine $81 \%$, and ciprofloxacine 85\%. Imipenem-, meropenem-, and amikacin-resistant strains were not detected in these patients.

Enterobacter spp. $(\mathrm{p}=0.534)$, streptococcus spp. $(\mathrm{p}=0.644)$, citrobacter $\mathrm{spp} .(\mathrm{p}=0.669)$, lactobacillus $(\mathrm{p}=0.406)$, and other microorganisms $(\mathrm{p}=0.283)$ did not differ according to the risk of growth in culture. Ampicillin resistance in Enterococci strains and methicillin resistance in Staphylococci strains were determined in cultures of risky patients. P. aeruginosa grew only in cultures of risky patients (Table 4). Antimicrobial susceptibility results of Gram-positive and -negative bacteria are presented in Tables 5 and 6. 
Of 20 patients with positive Staphylococcus culture, 16 (80\%) of them had different risk factors. The most common risk factors in these patients were biliary disease $(n=14,87.5 \%)$, $\mathrm{DM}(\mathrm{n}=5,31.2 \%)$, history of hospitalization during the previous year $(\mathrm{n}=3,18.7 \%$, and history of antibiotic use in the last three months $(n=2,12.5 \%)$. Polymicrobial microorganisms were isolated from 22 patients $(6.1 \%)$.

Eighty-one patients $(22.5 \%)$ underwent surgery while receiving treatment for cholecystitis, and the remaining 279 patients (77.5\%) received prophylactic antibiotics before surgery. Cefazolin was used in 254 patients $(91 \%)$ and ceftriaxone in 25 patients $(9 \%)$ for surgical prophylaxis. Prophylactic antibiotics were administered to $181(64.8 \%)$ patients $30 \mathrm{~min}$ before the incision. Ninety-one patients $(32.6 \%)$ received antibiotics $1 \mathrm{~h}$ before the incision, and seven $(2.5 \%)$ patients at the time of the incision.

\section{Discussion}

Cholecystectomy is one of the most common abdominal surgical procedures, and often performed due to acute cholecystitis and other complications of gallstones [11, 12]. Sufficient data concerning the bacteriology of bile in patients undergoing cholecystectomy for acute cholecystitis do not exist in the literature [13]. Previous studies reported that the rate of bile culture positivity ranged from $9 \%$ to $42 \%[14,16]$. In our study, the microorganisms were determined in $23.3 \%$ of the patients who underwent cholecystectomy, and our results were thought to be similar to previous studies.

E coli. Enterococcus spp., Klebsiella spp., and Enterobacter spp. are the most commonly identified microorganisms in bile culture $[13,14]$. These bacteria are typically found in the intestinal flora and migrate ascending through the duodenum to colonize the gallbladder. E. coli, Enterobacter spp., and Klebsiella spp. were the most frequently isolated factors in our non-risk patients.

Staphylococci were the most common bacteria in risky patients in our study. Staphylococcus spp. have grown in the bile culture in some studies, including the present study, even though it is not part of the enteric flora $[13,17]$. These bacteria are thought to possibly be of enteric origin due to changes in intestinal microbial flora and are less likely to reach the gallbladder via hematogenous route [17].

Gallbladder disease is known to occur, especially in middle-aged women [18]. Therefore, multiple studies on gallbladder cultures have shown a higher percentage of women patients in their cohorts $[17,18]$. The majority of the patients in our study were women, similar 
to the study conducted by Galili et al. [15]. In our study, culture positivity was found to be higher in men even though most of the study group consisted of women.

The study of Mahafzah et al. determined that advanced age and pre-operative endoscopic retrograde cholangiopancreatography $(\mathrm{ERCP})$ were significant risk factors for a positive bile culture [16]. Galili et al. demonstrated that advanced age, increased leukocyte count, high serum bilirubin, and high ALP levels accompanied bactobilia. The author in this study reported that the increase in laboratory values in patients with growth on the bile culture may be a sign of advanced gallbladder infection [15]. In our study, advanced age, history of biliary disease, undergoing biliary surgery before cholecystectomy, presence of biliary instrumentation, and an increase in leukocyte count, PNL ratio, CRP, creatinine, ALP, glucose levels, and total bilirubin values were found to be significant predictors of positive bile culture.

Capoor et al. demonstrated that $4.8 \%$ of the patients had polymicrobial infection in the gallbladder [19]. Yun et al. stated that two bacteria were identified together in the bile culture of $51.9 \%$ of the patients. In their study, they demonstrated that low BMI, previous ERCP, and presence of operative complications were significantly associated with multiple growth in bile culture [14]. In our study, polymicrobial growth was found in $6.1 \%$ of the patients. Although the results were not statistically significant, polymicrobial growth was higher in the risk group patients. Culture positivity and presence of resistant bacteria were found to be statistically significantly higher in the risk group patients.

Risk factors for multi-drug resistant bacteria were found to be DM, immunosuppression, and previous antibiotic therapy and hospitalization history. These risk factors should be evaluated while organizing the empirical treatment [8]. In a multi-center prospective study of patients with acute cholecystitis, 267 bacteria were isolated in the gallbladder culture of 567 patients, and $21(7.8 \%)$ of these bacteria were resistant. Furthermore, methicillin resistance was detected in two community-acquired S. aureus strains [13]. In our study, methicillin resistance in Staphylococci was determined only in the patients with risk factors.

Fluoroquinolones are frequently used in biliary tract infections due to its good diffusion to bile ducts and high oral bioavailability [20]. The guidelines for antimicrobial therapy for acute cholangitis and cholecystitis recommend fluoroquinolone therapy if the susceptibility of the strain grown in the culture is known or the patient has a beta lactam allergy [6]. Guidelines for the management of intra-abdominal infection state that fluoroquinolones can be used in the treatment of community-acquired low-risk intra-abdominal infections, but caution should be 
exercised in areas with high resistance [21]. In 2019, fluoroquinolone resistance was generally lower in the northern and western areas and higher in the southern and eastern areas of the WHO European Region. North Macedonia, the Republic of Moldova, the Russian Federation, and Turkey had resistance levels of $50 \%$ or higher [22]. In patients without any risk factors, fluoroquinolone sensitivity was determined as $85 \%$ in E coli strains in our study and it was concluded that fluoroquinolones should be used carefully in the selected patient group during administration of empirical treatment.

In the study conducted by Li et al. extended spectrum beta-lactamase (ESBL) positivity was determined as $39.5 \%$ in Klebsiella pneumonia strains isolated from bile samples [23]. In the study of Park et al., bile samples were obtained by endoscopic retrograde cholangiopancreatography and percutaneous transhepatic biliary drainage and demostrated the presence of E. coli and Klebsiella spp. These authours found that ESBL positivity in their strains was $31.1 \%$ in the initial cultures [3]. In our study, bile cultures were obtained preoperatively, and ESBL positivity was evaluated according to the presence of risk factors in our patients. Our study did not show that the ESBL resistance was statistically different in Gramnegative bacteria strains.

Administration of antimicrobial prophylaxis is recommended $60 \mathrm{~min}$ before surgical incision in patients undergoing either high risk laparoscopic or open cholecystectomy. Although antimicrobial prophylaxis is not recommended in low-risk patients, it is reported that it may be rational to give antimicrobial prophylaxis to all patients undergoing laparoscopic cholecystectomy since high risk cannot be determined before surgical intervention (24). Infection rates after laparoscopic cholecystectomy were reported to be $0 \%-7 \%$ and $0 \%-4 \%$ in patients with and without antimicrobial prophylaxis, respectively [24]. Our study showed that all patients who underwent biliary surgery were either under treatment or received applied surgical prophylaxis. Considering the increasing resistance rates, the need for surgical prophylaxis should be re-evaluated in patients without infection risk.

One limitation of our study is the small number of cases. However, we believe that the results are important because it is a national multicenter study. Another limitation of our study was the inability to perform metagenomic analyzes. Microbial culture has limitations and may miss several relevant microbes. However, it is possible to determine the microorganisms more comprehensively with metagenomic analysis [25]. Due to the high cost of metagenomic analysis and the lack of financial support, only preoperative specimen cultures and growth agent antibiotic susceptibility patterns could be investigated. 
It was determined in our study that the possibility of detecting the causative microorganism increases with male gender, > 60 years of age, history of biliary disease, underwent biliary surgery before cholecystectomy, having biliary instrumentation, operation concurrently performed with cholecystectomy, and presence of complication. It has been determined that patients may need a broader spectrum antibiotic if they have risk factors for developing resistant bacterial infections. Evaluation of anti-biotherapy according to individual risk factors in patients with acute cholecystitis will ensure the rational use of antibiotics.

\section{Availability of data and materials}

The datasets for the this study are available from the corresponding author on reasonable request

\section{Contributions to the paper}

All authors (D. Ozturk-Engin, C. Agalar, Y. Cag, F. Kesmez-Can, I. I. Balkan, O. Karabay, S. Senbayrak, B. Meral-Cetinkaya, M.T.Aydın, K. Tomas, E. Disci, A. Surmelioglu, O. Alimoglu, O. Ekinci, E. Akın, M. Köroğlu, M. Velidedeoglu, H. Ankaralı, E. Kocoglu, E. Ozmen, M. Javadov, B. Papilla-Kundaktepe, N. Oguzoglu, R. Donmez, E. Mega, S. Aksaray, F. Agalar ) have made significant contributions to the concept or design of the study. Material preparation, data collection and analysis were performed by these authors. They drafted and critically reviewed to the study. They have contributed to and are responsible for all aspects of his work. The final version of the article has been read and approved by all authors.

\section{Funding}

Supported by Infectious Diseases and Clinical Microbiology Speciality Society of Turkey.

\section{Ethical approval}

This study was performed in line with the principles of the Declaration of Helsinki. Approval was granted by the Ethics Committee of Fatih Sultan Mehmet Training and Research Hospital in Istanbul, Turkey.

\section{Conflicts of interest}

The authors have no conflicts of interest to declare that are relevant to the content of this article. All named authors have seen and reviewed the submitted version of the paper and agreed to all included data. 


\section{References}

1. Gupta E, Chakravarti A. Viral Infections of the Biliary Tract. Saudi J Gastroenterol. 2008; 14(3):158-160. https://doi.org/ 10.4103/1319-3767.41740.

2. Mustafa M, Menon J, Rahman MDS, et al. Acute Biliary tract infections, Diagnostic criteria and Treatment. Int J Pharm Sci Invent. 2014; 3(10):58-62.

https://doi.org/10.1016/j.jviscsurg. 2019.05.007.

3. Park JW, Lee JK, Lee KT, et al. How to interpret the bile culture results of patients with biliary tract infections. Clin Res Hepatol Gastroenterol. 2014;38 (3):300-9.

https://doi.org/10.1016/j.clinre.2014.02.005.

4. Maseda E, Maggi G, Gomez-Gil R, et al. Prevalence of and Risk Factors for Biliary Carriage of Bacteria Showing Worrisome and Unexpected Resistance Traits. J Clin Microbiol. 2013;51(2):518-521. https://doi.org/ 10.1128/JCM.02469-12.

5. Abeysuriya V, Deen KI, Wijesuriya $\mathrm{T}$, et al. Microbiology of gallbladder bile in uncomplicated symptomatic cholelithiasis. Hepatobiliary Pancreat Dis Int. 2008;7(6):633-637.

6. Gomi H, Solomkin JS, Schlossberg D, et al. Tokyo Guidelines 2018: antimicrobial therapy for acute cholangitis and cholecystitis. J Hepatobiliary Pancreat Sci. 2008;25(1):3-16. https://doi.org/ 10.1002/jhbp.518.

7. European Committee on Antimicrobial Susceptibility Testing (EUCAST) (2018): European Committee on Antimicrobial Susceptibility Testing breakpoint tables for interpretation of MICs and zone diameters, version 8.1.

8. Iribar BP, Peña EA, Rubio- Perez I. Not Just Complicated Cholecystitis-Beware of Risk Factors! Surg Infect Case Reports. 2017;2(1): 98-100. https://doi.org/ 10.1089/crsi.2017.0029.

9. Chow AW, Evans GA, Nathens AB, et al. Canadian practice guidelines for surgical intraabdominal infections. Can J Infect Dis Med Microbiol. 2010;21(1):11-37.

10. Adrian AI, Ian JB. Acute cholecystitis. BMJ 2002 :21; 325(7365): 639-643. https://doi.org/ 10.1136/bmj.325.7365.639.

11. Jaafar G, Hammarqvist F, Enochsson L, et al. Patient-related risk factors for postoperative infection after cholecystectomy. World J Surg. 2017;41(9):2240-2244.

https://doi.org/ 10.1007/s00268-017-4029-0 
12. Potts JR. What are the indications for cholecystectomy? Cleve Clin J Med. 1990; 57(1):407. https://doi.org/ 10.3949/ccjm.57.1.40.

13. Coccolini F, Sartelli M, Catena F, et al, CIAO and CIAOW study groups. Antibiotic resistance pattern and clinical out-comes in acute cholecystitis: 567 consecutive worldwide patientsin a prospective cohort study. Int J Surg. 2015;21:32-7. https://doi.org/10.1016/j.ijsu.2015.07.013.

14. Yun SP, Seo H-II. Clinical aspects of bile culture in patients undergoing laparoscopic cholecystectomy. Medicine (Baltimore). 2018;97(26): e11234.

https://doi.org/ 10.1097/MD.0000000000011234.

15. Galili O, Eldar S, Matter I, et al. The effect of bactibilia on the course and outcome of laparoscopic cholecystectomy. Eur J Clin Microbiol Infect Dis. 2008;27(9):797-803. https://doi.org/ 10.1007/s10096-008-0504-8.

16. Mahafzah AM, Daradkeh SS. Profile and predictors of bile infection in patients undergoing laparoscopic cholecystectomy. Saudi Med J. 2009;30(8):1044-8.

17.Darkahi B, Sandblom G, Liljeholm H, et al. Biliary Microflora in Patients Undergoing Cholecystectomy. Surg Infect. 2014;15(3):262-5. https://doi.org/ 10.1089/sur.2012.125.

18. Katyal A, Bala K, Bansal A, et al. Clinico-microbiological analysis of bactibilia isolates in patients of cholecystectomy. Int J Res Med Sci. 2017;5(9):4030-4035.

https://doi.org/10.18203/2320-6012.ijrms20173977.

19. Capoor MR, Nair D, Rajni, et al. Microflora of bile aspirates in patients with acute cholecystitis with or without cholelithiasis: a tropical experience. Braz J Infect Dis. 2008; 12(3):222-5. https://doi.org/ 10.1590/S1413-86702008000300012.

20. Zhanel GG, Walkty A, Vercaigne L, et al. The new fluoroquinolones: A critical review. Can J Infect Dis. 1999;10(3):207-38. https://doi.org/ 10.1155/1999/378394.

21. Mazuski JE, Tessier JM, May AK, et al. The Surgical Infection Society Revised Guidelines on the Management of Intra-Abdominal Infection. Surg Infect (Larchmt). 2017; 18(1):1-76. https://doi.org/10.1089/sur.2016.261.

22.https://www.euro.who.int/_data/assets/pdf_file/0003/469200/Central-Asian-andEuropean-Surveillance-of-Antimicrobial-Resistance.-Annual-report-2020-eng.pdf 
23. Li L, Zhu C, Huang H. Clinical epidemiology and outcomes of biliary tract infections caused by Klebsiella pneumoniae. Ann Transl Med. 2019;7(14): 304.

https://doi.org/ 10.21037/atm.2019.06.03

24. Clinical Practice Guidelines for Antimicrobial Prophylaxis in Surgery. ASHP Therapeutic Guidelines.

25. Dyrhovden R, Øvrebø KK, Nordahl MV, et al. Bacteria and fungi in acute cholecystitis. A prospective study comparing next generation sequencing to culture. J Infect. 2020;80(1):16-23. https://doi.org/ 10.1016/j.jinf.2019.09.015. 


\section{Supplementary Files}

This is a list of supplementary files associated with this preprint. Click to download.

- Tables.Bile.pdf 